\title{
Prevalence of Opportunistic Infections and Associated Factors among HIV Positive Patients taking Anti-Retroviral Therapy in DebreMarkos Referral Hospital, Northwest Ethiopia
}

\author{
Nurilign Abebe Moges* and Getachew Mullu Kassa \\ Nursing department, Medicine and Health Sciences College, DebreMarkos University, DebreMarkos, Ethiopia
}

\begin{abstract}
Introduction: Opportunistic infections (Ols) are infections that are more frequent or more severe because of immune-suppression in HIV-infected persons, and they are the major clinical manifestation of HIV patients. They indirectly affect the natural history of HIV disease. Many studies concentrated on drug adherence, survival and other aspects of the diseases but limited studies are evident on Ols after Anti-Retroviral Therapy (ART) initiated. So this study was conducted to assess the prevalence of opportunistic infections and associated factors among HIV positive patients taking (ART) in DebreMarkos referral hospital, Northwest Ethiopia.
\end{abstract}

Methods:A cross sectional study design was conducted among HIV patients taking ART from 5 to 7 years. The study was conducted in DebreMarkos referral hospital among 423 patients. The data was entered in EPI data version 3.1 and analysis with done by using Statistical Package for Social Sciences (SPSS) version 16.0. Bivariate and multivariate analysis was performed to determine the association of each independent variable with occurrence of Ols. $95 \% \mathrm{Cl}$ and $p$-value less than 0.05 were considered as significant association.

Result: Majority, 241(57\%) of the study participants were female patients. The mean age of the study participants was 35.17 , with standard deviation of $+/-9.481$ years. A total of 181 Ols were observed from the study participants during the study period. The commonest type of Ols were oral candidiasis $50(11.8 \%)$, followed by chronic diarrhea for greater than 1 month, 42(9.9\%) and tuberculosis, $41(9.7 \%)$. The factors associated with Ols were; Age less than 40 years old was protective $(A O R=0.47,95 \% \mathrm{Cl}=0.25,0.90)$; baseline WHO stages of III\&IV was risk for development of Ol by five times(AOR=4.759, $95 \% \mathrm{Cl}=2.163,10.469)$; those who used to chew khat was at risk of developing OI almost five times more likely than those who did not chew $(A O R=4.733,95 \% \mathrm{Cl}=1.185,18.915)$; patients with good ART adherence were less likely to develop Ol compare to their counter parts ( $A O R=0.163,95 \% \mathrm{Cl}=0.051,0.522)$; current hemoglobin level of $\geq 10 \mathrm{~g} / \mathrm{dl}$ was protective $(\mathrm{AOR}=0.313,95 \% \mathrm{Cl}=0.162,0.605)$; and recent weight of $<60 \mathrm{~kg}$ $(\mathrm{AOR}=3.658,95 \% \mathrm{Cl}=1.656,8.078)$.

Conclusion and recommendation: The prevalence of Ols among HIV patients on ART is still high namely oral candidiasis, pathogens that cause chronic diarrhea and tuberculosis. Education on adherence of medications, and use of prophylactic medications for Ols should be given for HIV patients taking ART. Similar studies should be conducted to identify the relation between substance abuse and Ols in HIV patients taking ART.

Keywords: Opportunistic infections; HIV Patients; Anti-retroviral therapy; DebreMarkos Referral Hospital; Ethiopia

\section{Introduction}

Opportunistic infections (OIs) are defined as infections that are more frequent or more severe because of immune-suppression in HIVinfected persons, and they are the major clinical manifestation of HIV patients [1,2].

The natural history of HIV disease may be indirectly affected by the occurrence of opportunistic diseases, because HIV viral load increases in patients with acute opportunistic diseases. Survival in people infected with HIV has improved because of an increasingly powerful array of antiretroviral treatments, but neurological symptoms due to co morbidity conditions still remains public health important for HIV infected individuals [3-5].

Severely immune-compromised HIV patients may develop a variety of opportunistic infections that have a significant impact on their well-being, quality of life, health care costs, and their survival [6].

The most common opportunistic diseases in HIV patients are Candida esophagitis, Pneumocystis carinii pneumonia (PCP), disseminated Mycobacterium avium complex (MAC) infection, cytomegalovirus (CMV), Cryptococcus, kaposi sarcoma, herpes zoster, and tuberculosis [2].

The risk for the development of OI in HIV patients depends on exposure to potential pathogens, virulence of the pathogens, the degree of host immunity, and the use of antimicrobial prophylaxis [6]. And majority of these OI are associated with an increased hazard of death in HIV patients. Patients experiencing morbidity from opportunistic diseases may have interruptions in antiretroviral therapy causing more rapid progression of HIV disease. In addition studies found that opportunistic infections cause an up regulation in HIV replication and higher viral loads [2,7-11].

${ }^{*}$ Corresponding author: Nurilign Abebe Moges, Nursing department, Medicine and Health Sciences College, DebreMarkos University, DebreMarkos, Ethiopia, Tel : +251-9-10-10-62-95; Fax : +251-58-771-17-64 ; E-mail: nure113@gmail.com

Received March 04, 2014; Accepted April 16, 2014; Published April 27, 2014

Citation: Moges NA, Kassa GM (2014) Prevalence of Opportunistic Infections and Associated Factors among HIV Positive Patients taking Anti-Retroviral Therapy in DebreMarkos Referral Hospital, Northwest Ethiopia. J AIDS Clin Res 5: 301. doi:10.4172/2155-6113.1000301

Copyright: ( 2014 Moges NA, et al. This is an open-access article distributed under the terms of the Creative Commons Attribution License, which permits unrestricted use, distribution, and reproduction in any medium, provided the original author and source are credited. 
Drug interactions may occur in patients who are taking antiretroviral therapy and other drugs for supportive care, treatment of opportunistic infections, and immunomodulation, as well as alternative drugs obtained from health care providers other than their primary provider [12].

OIs are one of the major causes of morbidity and mortality in patients with HIV infection throughout the world. Even if potent combination of antiretroviral therapy (ART) has reduced the incidence of OIs for certain patients with access to care, for those patients in the developed and developing world did not have access to care and have OIs $[13,14]$.

So this studywas conducted to assess the prevalence of OI and the associated factors for the development of OIs in HIV Positive Patients taking anti-retroviral therapy (ART) in DebreMarkos Referral Hospital, Northwest Ethiopia from 2005 to 2013.

\section{Methods}

\section{Study area and period}

The study was conducted in DebreMarkos Referral Hospital among ART followers in DebreMarkos town, Northwest Ethiopia. DebreMarkos is the capital city of East Gojjam Zone. It is located 300 $\mathrm{km}$ away from Addis Ababa, the capital city of Ethiopia, and $265 \mathrm{~km}$ from Bahir Dar capital city of Amhara regional state. The study was conducted from patients enrolled to treatment from September, 2005 to January, 2013 from registrations card.

\section{Study design}

Cross sectional study design was conducted.

\section{Study population}

All adult HIV positive individuals' record on care and support follow up who had started ART at DebreMarkos Referral Hospital ART clinic from September, 2005 to February, 2008 were traced to the year 2013 in the study.

\section{Sample size determination}

Since there was no similar study conducted in the study area, the prevalence of OIs among HIV patient on ART was considered to be $50 \% .95 \%$ confidence interval (CI) and $10 \%$ for patients with incomplete data was considered given a final sample size of 423 using single population proportion formula.

\section{Sampling technique}

A total 8412 patients were enrolled in ART starting from 2005 to 2013 but about 3290 of the patients started ART after 2008 and they were excluded from the study. The main reason patients excluded after 2008 entrance was to follow OI prevalence from patients with a minimum of 5 years and a maximum of 7 years. In addition, transfer out, lost, drops; children's $<15$ years old were also excluded since we did not have further information from these patients regarding their OI status and children were excluded because of the interest of the study was for adults only. Patients with initial OIs were not recorded as case for this study. Finally there were 1646 patients that fulfill the inclusion criteria from that a total of 423 were selected by using simple random sampling technique through computer generation number method using their identification number as sampling unit.

\section{Operational definition (taken from ART registration card)}

Lost to follow up: Not seen since $>/=1$ month $<3$ months.
Drop: Lost to follow up for $>3$ months.

Transfer out: A patient is referred to another health facility for care as evidenced by his/her document.

\section{Adherence}

Adherence is defined as good if adherence is $>95 \%(<2$ doses of 30 doses or $<3$ dose of 60 dose is missed) as documented by ART physician; poor if adherence is between $85 \%-94 \%$ (3-5 doses of 30 doses or 3-9 dose of 60 dose is missed) as documented by ART physician; as documented by ART physician.

\section{Opportunistic infections}

- Oesophageal candidiasis

- Kaposi's sarcoma

- Cryptococcal meningitis

- Oral recurrent candida

- Unexplained chronic diarrhoea $>1$ month

- Unexplained persistent fever $>1$ month

- Unexplained presumed weight loss $>10 \%$

- Pneumocystis jiroveci pneumonia

- Recurrent severe bacterial pneumonia

- Active TB

- Severe bacterial infection (pneumonia, empyema, pyomyosities, meningitis, suspected bacteraemia/septicemia, bone or joint infection)

- Others (anaemia, skin conditions, herpessimplex, toxoplasmosis)

\section{Data processing and analysis}

The data was entered in EPI data version 3.1 and analysis was done by using Statistical Package for Social Sciences (SPSS) version 16.0. Prior to the analysis, the whole data was cleaned, coded and entered. The completeness of the data was checked. Bivariate analysis was performed to determine the association of each independent variable with the dependent variable (occurrence of OIs). Variables with P-value less than 0.25 and known associated factors from different literature were included for further analysis using the multivariate analysis. 95\% $\mathrm{CI}$ and $\mathrm{p}$-value less than 0.05 considered as significant association.

\section{Ethical consideration}

Ethical clearance was obtained from Institutional Research Ethics Review Committee (IRERC) of DebreMarkos University, College of Medicine and Health science (Ref. No: HSC/1210/21/05). Then officials at different levels in the study area were communicated through letters from department of public health. Permission was obtained from DebreMarkos Referral Hospital and ART clinic. Confidentiality of the information was assured and privacy of the information was also maintained.

\section{Results}

This study assessed the prevalence of and associated factors for OIs. The study included 423 patients HIV Positive Patients who were in ART from 5 to 7 years in DebreMarkos Referral Hospital. 
Majority, 241(57\%) of the study participants were female patients. The mean age of the study participants was 35.17 , with standard deviation of $+/-9.48$ years. More than two third, 392(92.7\%) of patients were Orthodox religion followers, and more than half, 239(56.5\%) of patients were not employed in any governmental and/or Nongovernmental organizations. When classified by occupation majority, $89(21 \%)$ of the respondents were government employees, followed by merchant, 87(20.6\%), daily laborer, 79(18.7\%), and farmer, 74(17.5\%). Majority, $172(40.7 \%)$ of patients were married while $43(4.2 \%)$ were never married. One hundred twenty two $(28.8 \%)$ of patients didn't follow any formal education, while $48(11.3 \%)$ were followed college or/and University studies. Most of, 297(70.2\%) of the study participants residence were urban (Table 1).

\section{Prevalence of opportunistic infections}

A total of 181 OIs were observed from the study participants during the study period. The overall prevalence of OIs among the study participants were $42.8 \%$. From these; $86(61 \%)$ of patients had only 1 opportunistic infection during study period, while the prevalence of 2 , 3 , and 4 OIs per one patient during the study period were $49(34.8 \%)$, $5(3.5 \%)$, and $1(0.7 \%)$ respectively (Table 2$)$. The commonest type of OIs among HIV patients in ART in the current study area were oral candidiasis $50(11.8 \%)$, followed by chronic diarrhea for greater than 1 month $42(9.9 \%)$ and tuberculosis $41(9.7 \%)$. The prevalence

\begin{tabular}{|l|c|c|}
\hline Characteristics & Frequency & Percentage \\
\hline Sex & 182 & 43 \\
Male & 241 & 57 \\
\hline Female & & \\
\hline Age & 325 & 76.8 \\
\hline < 4o years old & 98 & 23.2 \\
\hline$\geq 40$ years old & & \\
\hline Religion & 392 & 92.7 \\
Orthodox & 6 & 1.4 \\
Protestant & 24 & 5.7 \\
Muslim & 1 & 0.2 \\
\hline Others & 184 & \\
\hline Occupation & 239 & 43.5 \\
Employed & & 56.5 \\
\hline Not employed & 172 & \\
\hline Marital status & 88 & 40.7 \\
Married & 43 & 20.8 \\
\hline Widowed & 101 & 10.2 \\
Never married & 19 & 23.9 \\
\hline Divorced & & 4.5 \\
\hline Separated & 122 & \\
\hline Educational status & 148 & 28.8 \\
\hline No education & 105 & 35.0 \\
Primary & 48 & 24.8 \\
Secondary & 126 & 11.3 \\
\hline Tertiary & 297 & 29.8 \\
\hline Place of residence & & 70.2 \\
\hline Rural & & \\
\hline Urban & & \\
\hline Table & & \\
\hline
\end{tabular}

Table 1: Socio demographic characteristics of HIV patients taking Anti-Retroviral Therapy in Debre Markos Referral Hospital, Northwest Ethiopia; 2013 ( $n=423)$.

\begin{tabular}{|c|c|c|c|}
\hline S.No. & Opportunistic infection & Frequency & Percentage \\
\hline 1. & 1 opportunistic infection & 86 & 61 \\
\hline 2. & 2 opportunistic infections & 49 & 34.8 \\
\hline 3. & 3 opportunistic infections & 5 & 3.5 \\
\hline 4. & 4 Opportunistic Infections & 1 & 0.7 \\
\hline & Total & 141 & 100 \\
\hline
\end{tabular}

Table 2: Number of Opportunistic Infections diagnosed per patient among HIV patients taking Anti-Retroviral Therapy in Debre Markos Referral Hospital, Northwest Ethiopia; 2013.

\begin{tabular}{|c|l|c|c|}
\hline S.No. & Opportunistic infection & Frequency & Percentage \\
\hline 1 & Oral candidiasis & 50 & 11.8 \\
\hline 2 & Chronic diarrhea (>1 month) & 42 & 9.9 \\
\hline 3 & Tuberculosis & 41 & 9.7 \\
\hline 4 & Esophageal candiasis & 19 & 4.5 \\
\hline 5 & Severe bacterial pneumonia & 13 & 3.1 \\
\hline 6 & Pneumocystic pneumonia & 12 & 2.8 \\
\hline 7 & Cryptococcus meningitis & 2 & 0.5 \\
\hline 8 & Septicemia & 2 & 0.5 \\
\hline
\end{tabular}

Table 3: Prevalence of Opportunistic infections among HIV patients taking Anti Retroviral Therapy in Debre Markos Referral Hospital, Northwest Ethiopia; 2013.

of Esophageal candidiasis was 19(4.5\%), Cryptococcus meningitis 2(0.5\%), Pneumocystic pneumonia 12(2.8\%), septicemia 2(0.5\%) and sever bacterial pneumonia 13(3.1\%) (Table 3).

\section{Factors associated with OIs among HIV patients taking ART}

Bivariate and multivariate analysis was conducted to identify the associated factors for occurrence of OIs among HIV patient taking ART. As described in Table 4; the independent variables were found to have association with occurrence of OIs in bivariate analysis were age less than 40 years old were 0.47 times less likely to acquire OI than patients at older ages $(\mathrm{COR}=0.47,95 \% \mathrm{CI}=0.29,0.74)$, educational status of primary education was preventive compared to non-educated patients $(\mathrm{COR}=0.590,95 \% \mathrm{CI}=0.356,0.978)$, patients at baseline WHO stages III\&IV were at high risk of OI development after ART initiation too, $(\mathrm{COR}=6.343,95 \% \mathrm{CI}=3.183,12.64)$, patients who used to chew Khat was found to be at risk for OI occurrence $(\mathrm{COR}=3.00$, $95 \% \mathrm{CI}=1.12,8.06$ ), good ART adherence was preventive for OI occurrence $(\mathrm{COR}=0.20,95 \% \mathrm{CI}=0.09,0.45)$, and patients with a base line CD4 count of less than 200 cells/ $\mu$ l were almost double risk of OI development than their counter parts with higher $\mathrm{CD} 4$ count at base line $(\mathrm{COR}=1.91,95 \% \mathrm{CI}=1.16,3.14)$.

Variables with a p-value of less than 0.25 from the bivariate analysis and variables with $p>0.25$ but found to have association with occurrence of OIs among HIV patient taking ART from similar study, were entered to multivariate analysis to identify the significant associated factors for OIs. Accordingly, as described in Table 5, the associated factors for OIs among HIV patient taking ART at p-value less than 0.05 were; Age less than 40 years old were preventive for OI occurrence (AOR $=0.47$, $95 \% \mathrm{CI}=0.25,0.90)$; baseline $\mathrm{WHO}$ stages III\&IV were almost five times at risk of $\mathrm{OI}(\mathrm{AOR}=4.76,95 \% \mathrm{CI}=2.16,10.47)$; patients whose record show history of khat use were 4.73 times more likely to be affected by different OIs $(\mathrm{AOR}=4.73,95 \% \mathrm{CI}=1.19,18.92)$; patients with good ART adherence were 0.16 times less likely to develop $\mathrm{OI}(\mathrm{AOR}=0.16$, $95 \% \mathrm{CI}=0.05,0.52)$; patients with recent hemoglobin level of $\geq 10 \mathrm{~g} / \mathrm{dl}$ were 0.31 times less likely to develop OI after ART started $(\mathrm{AOR}=0.31$, $95 \% \mathrm{CI}=0.162,0.61)$; and patients with recent weight of $<60 \mathrm{~kg}$ were at higher risk of developing OIs than their counter parts with greater than $60 \mathrm{~kg}(\mathrm{AOR}=3.66,95 \% \mathrm{CI}=1.66,8.08)$.

\section{Discussion}

The current study assessed the prevalence and associated factors of opportunistic infections among HIV Positive Patients. The study found that $141(33.3 \%)$ of HIV patients taking ART had got at least one OI during the study period and overall OIs prevalence of $42.8 \%$ with repeated infection. When compared with other similar studies, the prevalence of OIs in the current study area is comparable with a study conducted by Manosuthi et al. in a resource limited setting with a prevalence of $30 \%$ [15]. 
Citation: Moges NA, Kassa GM (2014) Prevalence of Opportunistic Infections and Associated Factors among HIV Positive Patients taking AntiRetroviral Therapy in DebreMarkos Referral Hospital, Northwest Ethiopia. J AIDS Clin Res 5: 301. doi:10.4172/2155-6113.1000301

Page 4 of 6

\begin{tabular}{|c|c|c|c|c|c|c|c|}
\hline \multirow{2}{*}{ S.No } & \multirow{2}{*}{ Variables } & \multicolumn{2}{|c|}{ Opportunistic Infection } & \multirow{2}{*}{ COR } & \multicolumn{2}{|c|}{$95 \% \mathrm{Cl}$} & \multirow{2}{*}{ P-value } \\
\hline & & Absent & Present & & Lower & Upper & \\
\hline 1. & $\begin{array}{l}\text { Sex } \\
\text { Male } \\
\text { Female }\end{array}$ & $\begin{array}{l}127(69.8 \%) \\
155(64.3 \%\end{array}$ & $\begin{array}{l}55(30.2 \%) \\
86(35.7 \%)\end{array}$ & 0.781 & $\begin{array}{c}0.517 \\
1\end{array}$ & 1.178 & 0.238 \\
\hline 2. & $\begin{array}{l}\text { Age } \\
<40 \text { years old } \\
\geq 40 \text { years old }\end{array}$ & $\begin{array}{c}230(70.8 \%) \\
52(53.1 \%)\end{array}$ & $\begin{array}{l}95(29.2 \%) \\
46(46.9 \%)\end{array}$ & 0.467 & $\begin{array}{c}0.294 \\
1\end{array}$ & 0.742 & $0.001^{*}$ \\
\hline 3. & $\begin{array}{l}\text { Educational status } \\
\text { No education } \\
\text { Primary } \\
\text { Secondary } \\
\text { Tertiary }\end{array}$ & $\begin{array}{c}72(59.0 \%) \\
105(70.9 \%) \\
73(69.5 \%) \\
32(66.7 \%)\end{array}$ & $\begin{array}{l}50(41.0 \%) \\
43(29.1 \%) \\
32(30.5 \%) \\
16(33.3 \%)\end{array}$ & $\begin{array}{l}0.590 \\
0.631 \\
0.720\end{array}$ & $\begin{array}{c}1 \\
0.356 \\
0.364 \\
0.357\end{array}$ & $\begin{array}{l}0.978 \\
1.094 \\
1.450\end{array}$ & $\begin{array}{l}\mathbf{0 . 0 4 1}^{*} \\
0.101 \\
0.358\end{array}$ \\
\hline 4. & $\begin{array}{l}\text { Place of residence } \\
\text { Rural } \\
\text { Urban }\end{array}$ & $\begin{array}{c}83(65.9 \%) \\
199(67.0 \%)\end{array}$ & $\begin{array}{l}43(34.1 \%) \\
98(33.0 \%)\end{array}$ & 1.052 & $\begin{array}{c}0.677 \\
1\end{array}$ & 1.634 & 0.822 \\
\hline 5. & $\begin{array}{l}\text { Baseline WHO stage } \\
\text { Stage I/II } \\
\text { Stage III/IV }\end{array}$ & $\begin{array}{c}92(90.2 \%) \\
190(59.2 \%)\end{array}$ & $\begin{array}{c}10(9.8 \%) \\
131(40.8 \%)\end{array}$ & $\begin{array}{c}1 \\
6.343\end{array}$ & 3.183 & 12.64 & $0.000^{*}$ \\
\hline 6. & $\begin{array}{l}\text { Alcohol use } \\
\text { Yes } \\
\text { No }\end{array}$ & $\begin{array}{l}107(62.9 \%) \\
175(69.2 \%)\end{array}$ & $\begin{array}{l}63(37.1 \%) \\
78(30.8 \%)\end{array}$ & 1.436 & $\begin{array}{c}0.819 \\
1\end{array}$ & 2.517 & 0.207 \\
\hline 7. & $\begin{array}{l}\text { Cigarrate smoking } \\
\text { Yes } \\
\text { No }\end{array}$ & $\begin{array}{c}10(55.6 \%) \\
272(67.2 \%)\end{array}$ & $\begin{array}{c}8(44.4 \%) \\
133(32.8 \%)\end{array}$ & 1.109 & $\begin{array}{c}0.304 \\
1\end{array}$ & 4.045 & 0.875 \\
\hline 8. & $\begin{array}{l}\text { khat use } \\
\text { Yes } \\
\text { No }\end{array}$ & $\begin{array}{c}7(41.2 \%) \\
275(67.7 \%)\end{array}$ & $\begin{array}{c}10(58.8 \%) \\
131(32.3 \%)\end{array}$ & 2.999 & $\begin{array}{c}1.117 \\
1\end{array}$ & 8.055 & $0.029^{*}$ \\
\hline 9. & $\begin{array}{l}\text { ART adherence } \\
\text { Good } \\
\text { Poor }\end{array}$ & $\begin{array}{c}273(69.3 \%) \\
9(31.0 \%)\end{array}$ & $\begin{array}{c}121(30.7 \%) \\
20(69.0 \%)\end{array}$ & 0.199 & $\begin{array}{c}0.088 \\
1\end{array}$ & 0.451 & $0.000^{*}$ \\
\hline 10. & $\begin{array}{l}\text { Occupation } \\
\text { Employed } \\
\text { Not employed }\end{array}$ & $\begin{array}{l}127(69.0 \%) \\
155(64.9 \%)\end{array}$ & $\begin{array}{l}57(31.0 \%) \\
84(35.1 \%)\end{array}$ & 0.828 & $\begin{array}{c}0.550 \\
1\end{array}$ & 1.248 & 0.368 \\
\hline 11. & $\begin{array}{l}\text { Baseline CD4 count } \\
<200 \\
200\end{array}$ & $\begin{array}{c}43(54.4 \%) \\
239(69.5 \%)\end{array}$ & $\begin{array}{c}36(45.6 \%) \\
105(30.5 \%)\end{array}$ & 1.906 & $\begin{array}{c}1.157 \\
1\end{array}$ & 3.138 & $0.011^{*}$ \\
\hline 12. & $\begin{array}{l}\text { Current Hemoglobin level } \\
<10 \\
\geq 10\end{array}$ & $\begin{array}{c}23(36.5 \%) \\
201(71.8 \%)\end{array}$ & $\begin{array}{l}40(63.5 \%) \\
79(28.2 \%)\end{array}$ & 0.295 & $\begin{array}{c}1 \\
0.024\end{array}$ & 3.596 & 0.339 \\
\hline 13. & $\begin{array}{l}\text { Marital status } \\
\text { Single** } \\
\text { Married }\end{array}$ & $\begin{array}{l}159(63.3 \%) \\
123(71.5 \%)\end{array}$ & $\begin{array}{l}92(36.7 \%) \\
49(28.5 \%)\end{array}$ & 1.452 & $\begin{array}{c}0.955 \\
1\end{array}$ & 2.208 & 0.081 \\
\hline 14. & $\begin{array}{l}\text { Ol prophylaxis given }{ }^{* * *} \\
\text { Yes } \\
\text { No }\end{array}$ & $\begin{array}{c}25(55.6 \%) \\
257(68.0 \%)\end{array}$ & $\begin{array}{c}20(44.4 \%) \\
121(32.0 \%)\end{array}$ & 1.699 & $\begin{array}{c}0.908 \\
1\end{array}$ & 3.179 & 0.097 \\
\hline 15. & $\begin{array}{l}\text { Condom use } \\
\text { Yes } \\
\text { No }\end{array}$ & $\begin{array}{c}238(66.3 \%) \\
44(68.8 \%)\end{array}$ & $\begin{array}{c}121(33.7 \%) \\
20(31.2 \%)\end{array}$ & 1.118 & $\begin{array}{c}0.631 \\
1\end{array}$ & 1.982 & 0.701 \\
\hline 16. & $\begin{array}{l}\text { Recent weight } \\
<60 \mathrm{~kg} \\
\geq 60 \mathrm{~kg}\end{array}$ & $\begin{array}{c}208(61.9 \%) \\
74(85.1 \%)\end{array}$ & $\begin{array}{c}128(38.1 \%) \\
13(14.9 \%)\end{array}$ & 3.503 & $\begin{array}{c}1.867 \\
1\end{array}$ & 6.572 & $0.000^{*}$ \\
\hline
\end{tabular}

*Significant association at $95 \% \mathrm{Cl},{ }^{* *}$ Single includes: Widowed, Never married, Divorced and Separated ${ }^{* * *}$ Ol prophylaxis include: Cotrmoxazole and/or INH and/or Fluconazole

Table 4: Bivariate analysis variables with occurrence of opportunistic Infections among HIV Positive Patients taking Anti-Retroviral Therapy in Debre Markos Referral Hospital, Northwest Ethiopia; 2013.

A study conducted in Taiwan showed that the prevalence of OIs to be $47.6 \%$, which is greater than the current study area [16]. The reason for such difference could be due to the difference in the duration of follow up. The current study followed the study participant for 5 to 7 years while the earlier was for shorter period that may give less chance for observation of OIs. Other study conducted in Nigeria also showed that OI prevalence to be $22.4 \%$, which is below the result for the current study. The reason for such difference could be due to the difference in the socio demographic characteristics, most of study participants in this study were at advanced HIV stage and sample size difference. The current study used a sample of 423, while the study in Nigeria used 354 [17].
From different OIs in HIV patients, the common types of OIs among HIV patients on ART in the current study area were oral candidacies, chronic diarrhea for more than 1 month and tuberculosis. Similar findings were also observed in other studies [16-18]. This might be because of their diagnosis is relatively easy to identify from patients than other OIs.

Factorsassociated with occurrence of OIs among HIV patients taking ART were also assessed. Accordingly younger age, advanced base line WHO stage, khat use, ART adherence, recent hemoglobin status, and recent weight were found to be associated factor for OIs occurrence. 
Citation: Moges NA, Kassa GM (2014) Prevalence of Opportunistic Infections and Associated Factors among HIV Positive Patients taking AntiRetroviral Therapy in DebreMarkos Referral Hospital, Northwest Ethiopia. J AIDS Clin Res 5: 301. doi:10.4172/2155-6113.1000301

Page 5 of 6

\begin{tabular}{|c|c|c|c|c|c|c|c|}
\hline \multirow{2}{*}{ S.No } & \multirow{2}{*}{ Variables } & \multicolumn{2}{|c|}{ Opportunistic Infection } & \multirow{2}{*}{ COR } & \multicolumn{2}{|c|}{$95 \% \mathrm{Cl}$} & \multirow{2}{*}{$P$-value } \\
\hline & & Absent & Present & & Lower & Upper & \\
\hline 1. & $\begin{array}{l}\text { Sex } \\
\text { Male } \\
\text { Female }\end{array}$ & $\begin{array}{c}127(69.8 \%) \\
155(64.3 \%\end{array}$ & $\begin{array}{l}55(30.2 \%) \\
86(35.7 \%)\end{array}$ & 0.814 & $\begin{array}{c}0.442 \\
1\end{array}$ & 1.498 & 0.508 \\
\hline 2. & $\begin{array}{l}\text { Age } \\
<40 \text { years old } \\
\geq 40 \text { years old }\end{array}$ & $\begin{array}{c}230(70.8 \%) \\
52(53.1 \%)\end{array}$ & $\begin{array}{l}95(29.2 \%) \\
46(46.9 \%)\end{array}$ & 0.471 & $\begin{array}{c}0.247 \\
1\end{array}$ & 0.896 & $0.022^{*}$ \\
\hline 3. & $\begin{array}{l}\text { Educational status } \\
\text { No education } \\
\text { Primary } \\
\text { Secondary } \\
\text { Tertiary }\end{array}$ & $\begin{array}{c}72(59.0 \%) \\
105(70.9 \%) \\
73(69.5 \%) \\
32(66.7 \%)\end{array}$ & $\begin{array}{l}50(41.0 \%) \\
43(29.1 \%) \\
32(30.5 \%) \\
16(33.3 \%)\end{array}$ & $\begin{array}{l}0.809 \\
1.144 \\
1.119\end{array}$ & $\begin{array}{c}1 \\
0.402 \\
0.529 \\
0.361\end{array}$ & $\begin{array}{l}1.630 \\
2.471 \\
3.469\end{array}$ & $\begin{array}{l}0.553 \\
0.733 \\
0.846\end{array}$ \\
\hline 4. & $\begin{array}{l}\text { Place of residence } \\
\text { Rural } \\
\text { Urban }\end{array}$ & $\begin{array}{c}83(65.9 \%) \\
199(67.0 \%)\end{array}$ & $\begin{array}{l}43(34.1 \%) \\
98(33.0 \%)\end{array}$ & 0.956 & $\begin{array}{c}0.513 \\
1\end{array}$ & 1.782 & 0.888 \\
\hline 5. & $\begin{array}{l}\text { Baseline WHO stage } \\
\text { Stage I-II } \\
\text { Stage III-IV }\end{array}$ & $\begin{array}{c}92(90.2 \%) \\
190(59.2 \%)\end{array}$ & $\begin{array}{c}10(9.8 \%) \\
131(40.8 \%)\end{array}$ & 4.759 & $\begin{array}{c}1 \\
2.163\end{array}$ & 10.469 & $0.000^{*}$ \\
\hline 6. & $\begin{array}{l}\text { Alcohol use } \\
\text { Yes } \\
\text { No }\end{array}$ & $\begin{array}{l}107(62.9 \%) \\
175(69.2 \%)\end{array}$ & $\begin{array}{l}63(37.1 \%) \\
78(30.8 \%)\end{array}$ & 1.436 & $\begin{array}{c}0.819 \\
1\end{array}$ & 2.517 & 0.207 \\
\hline 7. & $\begin{array}{l}\text { Cigarrate smoking } \\
\text { Yes } \\
\text { No }\end{array}$ & $\begin{array}{c}10(55.6 \%) \\
272(67.2 \%)\end{array}$ & $\begin{array}{c}8(44.4 \%) \\
133(32.8 \%)\end{array}$ & 1.109 & $\begin{array}{c}0.304 \\
1\end{array}$ & 4.045 & 0.875 \\
\hline 8. & $\begin{array}{l}\text { Khat use } \\
\text { Yes } \\
\text { No }\end{array}$ & $\begin{array}{c}7(41.2 \%) \\
275(67.7 \%)\end{array}$ & $\begin{array}{c}10(58.8 \%) \\
131(32.3 \%)\end{array}$ & 4.733 & $\begin{array}{c}1.185 \\
1\end{array}$ & 18.915 & $0.028^{*}$ \\
\hline 9. & $\begin{array}{l}\text { ART adherence } \\
\text { Good } \\
\text { Poor }\end{array}$ & $\begin{array}{c}273(69.3 \%) \\
9(31.0 \%)\end{array}$ & $\begin{array}{c}121(30.7 \%) \\
20(69.0 \%)\end{array}$ & 0.163 & 0.0511 & 0.522 & $0.002^{*}$ \\
\hline 10. & $\begin{array}{l}\text { Occupation } \\
\text { Employed } \\
\text { Not employed }\end{array}$ & $\begin{array}{l}127(69.0 \%) \\
155(64.9 \%)\end{array}$ & $\begin{array}{l}57(31.0 \%) \\
84(35.1 \%)\end{array}$ & 0.974 & $\begin{array}{c}0.521 \\
1\end{array}$ & 1.820 & 0.934 \\
\hline 11. & $\begin{array}{l}\text { Baseline CD4 count } \\
<200 \\
\geq 200\end{array}$ & $\begin{array}{c}43(54.4 \%) \\
239(69.5 \%)\end{array}$ & $\begin{array}{c}36(45.6 \%) \\
105(30.5 \%)\end{array}$ & 1.340 & $\begin{array}{c}0.678 \\
1\end{array}$ & 2.649 & 0.400 \\
\hline 12. & $\begin{array}{l}\text { Current Hemoglobin level } \\
<10 \\
>10\end{array}$ & $\begin{array}{c}23(36.5 \%) \\
201(71.8 \%)\end{array}$ & $\begin{array}{l}40(63.5 \%) \\
79(28.2 \%)\end{array}$ & 0.313 & $\begin{array}{c}1 \\
0.162\end{array}$ & 0.605 & $0.001^{*}$ \\
\hline 13. & $\begin{array}{l}\text { Marital status } \\
\text { Single } \\
\text { Married }\end{array}$ & $\begin{array}{l}159(63.3 \%) \\
123(71.5 \%)\end{array}$ & $\begin{array}{l}92(36.7 \%) \\
49(28.5 \%)\end{array}$ & 0.700 & $\begin{array}{c}0.398 \\
1\end{array}$ & 1.230 & 0.215 \\
\hline 14. & $\begin{array}{l}\text { Ol prophylaxis given }{ }^{* * *} \\
\text { Yes } \\
\text { No }\end{array}$ & $\begin{array}{c}25(55.6 \%) \\
257(68.0 \%)\end{array}$ & $\begin{array}{c}20(44.4 \%) \\
121(32.0 \%)\end{array}$ & 2.186 & $\begin{array}{c}0.937 \\
1\end{array}$ & 5.102 & 0.070 \\
\hline 15. & $\begin{array}{l}\text { Condom use } \\
\text { Yes } \\
\text { No }\end{array}$ & $\begin{array}{c}238(66.3 \%) \\
44(68.8 \%)\end{array}$ & $\begin{array}{c}121(33.7 \%) \\
20(31.2 \%)\end{array}$ & 1.449 & $\begin{array}{c}0.676 \\
1\end{array}$ & 3.102 & 0.340 \\
\hline 16. & $\begin{array}{l}\text { Recent weight } \\
<60 \mathrm{~kg} \\
\geq 60 \mathrm{~kg}\end{array}$ & $\begin{array}{c}208(61.9 \%) \\
74(85.1 \%)\end{array}$ & $\begin{array}{c}128(38.1 \%) \\
13(14.9 \%)\end{array}$ & 3.658 & $\begin{array}{c}1.656 \\
1\end{array}$ & 8.078 & $0.001^{*}$ \\
\hline
\end{tabular}

*Significant association at $95 \% \mathrm{Cl}$, ${ }^{* *}$ Single includes: Widowed, Never married, Divorced and Separated ${ }^{* * *}$ Ol prophylaxis include: Cotrmoxazole and/or INH and/or Fluconazole

Table 5: Multivariate analysis for selected variables with occurrence of opportunistic Infections among HIV Positive Patients taking Anti-Retroviral Therapy in Debre Markos Referral Hospital, Northwest Ethiopia; 2013

Patients with age less than 40 year were less likely to develop OIs than their counter parts. A study conducted in India also found that being old people as a predominant factor for OIs [19]. This could be explained by older patients immune get decreased while the viral load gets increased that predispose for opportunistic infections which is not otherwise cause for younger patients in normal condition.

In addition, patients with advanced baseline WHO stages of III \& IV were more likely to develop OIs by a factor of 4.8 , than those with a base line WHO stage of I \& II. Similar finding were also observed in a study conducted in Nigeria showing that advanced WHO clinical stage at baseline to be an independent clinical risk factors for the occurrence of OIs [17]. Similar findings were also observed in studies [15,20-23]. This could be because of lower immunity as WHO staging is higher that further predispose for OIs. Majority of Patients also start treatment at their advanced stage of AIDS that will be difficult to reverse the stage easily with treatment.

Peoples who use khat along with ART medications are at risk to develop OIs by 4.733 than those who don't use Khat. Alcohol use and 
cigarette smoking were not found to be associated with occurrence of OIs among HIV patients taking ART. Similar study conducted in resource limited setting in Nigeria also found alcohol consumption and smoking to have no association with occurrence of OIs among HIV patients taking ART [17]. Due to inadequate studies conducted on a relationship between substance abuse and OIs among HIV patients taking ART medication, further study is recommended to determine the effect of substance abuse on clinical and immunologic status of peoples living with HIV (PLWH).

ART drug adherence was also found to have a significant association with occurrence of OIs. Peoples with good ART adherence were less likely to develop OIs on the course of HIV infection, while HIV patients with poor ART adherence were $74 \%$ time more likely to get OIs. This finding was also similar with other studies $[17,24,25]$. This might be because of the current study also found that $93.1 \%$ of PLWH have good adherence to ART medications. This is higher when compared with adherence of $78 \%$ in a study conducted in Nigeria [17]. Another explaining reason could be good adherence for ART will suppress viral replication and would increase $\mathrm{CD} 4$ cells that intern decrease risk of new opportunistic infection.

HIV Patients with a recent weight of less than $60 \mathrm{~kg}$ were 3.7 times more likely to develop OIs than their counter parts. In addition, current hemoglobin status of patients was also another factor found to be associated with occurrence of OIs. Patients with a current hemoglobin level of greater than $10 \mathrm{mg} / \mathrm{dl}$ were less likely to develop OIs while, patients with hemoglobin level of less than $10 \mathrm{mg} / \mathrm{dl}$ were $84 \%$ times more likely to develop OIs. Similar studies conducted in Nigeria also support this idea [17].

\section{Limitations of the study}

Using secondary data in which some important variables were not documented well and many opportunistic infections was presumed diagnosis may be mentioned as the possible limitations of this study.

\section{Concluding Remarks}

This study showed the prevalence of OIs among HIV patients on ART is still high, which requires several activities from the ministry of health $(\mathrm{MOH})$, hospitals, health professionals and patients. The common types of OIs among HIV patients taking ART are oral candidacies, chronic diarrhea and tuberculosis.factors associated with OIs occurrence were age less than 40 years, advanced base line WHO stage, khat use, ART adherence, current hemoglobin status, and recent weight. For PLWH who are on HAART, health professional should give necessary education on adherence of medications, and use of prophylactic medications to prevent OIs. Studies should also be conducted to identify the relation between substance abuse and OIs in HIV patients taking ART and further follow up study will help to identify OIs occurrence.

\section{References}

1. CDC (2009) Guidelines for Prevention and Treatment of Opportunistic Infections in HIV-Infected Adults and Adolescents. Recommendations and Reports 58.

2. Chaisson RE, Gallant JE, Keruly JC, Moore RD (1998) Impact of opportunistic disease on survival in patients with HIV infection. AIDS 12: 29-33.

3. Donovan RM, Bush CE, Markowitz NP, Baxa DM, Saravolatz LD (1996) Changes in virus load markers during AIDS-associated opportunistic diseases in human immunodeficiency virus-infected persons. J Infect Dis 174: 401-403.

4. Goletti D, Weissman D, Jackson RW, Graham NM, Vlahov D, et al. (1996) Effect of Mycobacterium tuberculosis on HIV replication. Role of immune activation. J Immunol 157: 1271-1278.

5. Tan IL, Smith BR, von Geldern G, Mateen FJ, McArthur JC (2012) HIVassociated opportunistic infections of the CNS. Lancet Neurol 11: 605-617.
6. Chaisson RE, Moore RD (1997) Prevention of opportunistic infections in the era of improved antiretroviral therapy. J Acquir Immune DeficSyndr Hum Retrovirol 16: S14-22.

7. Glesby MJ, Hoover DR, Farzadegan H, Margolick JB, Saah AJ (1996) The effect of influenza vaccination on human immunodeficiency virus type 1 load: a randomized, double-blind, placebo-controlled study. J Infect Dis 174: 13321336.

8. Palacios R, Jiménez-Oñate F, Aguilar M, Galindo MJ, Rivas P, et al. (2007) Impact of syphilis infection on HIV viral load and CD4 cell counts in HIV-infected patients. J Acquir Immune DeficSyndr 44: 356-359.

9. Bentwich Z, Maartens G, Torten D, Lal AA, Lal RB (2000) Concurrent infections and HIV pathogenesis. AIDS 14: 2071-2081.

10. Sulkowski MS, Chaisson RE, Karp CL, Moore RD, Margolick JB, et al. (1998) The effect of acute infections illnesses on plasma human immunodeficiency virus (HIV) type 1 load and the expression of serologic markers of immune activation among HIV-infected adults. J Infect Dis 178: 1642-1648.

11. Donovan RM, Bush CE, Markowitz NP, Baxa DM, Saravolatz LD (1996) Changes in Virus Load Markers during AIDS-Associated Opportunistic Diseases in Human Immuno deficiency Virus-Infected Persons. J Infect Dis 174: 401-403.

12. Piscitelli SC, Gallicano KD (2001) Interactions among drugs for HIV and opportunistic infections. N Engl J Med 344: 984-996.

13. Soriano V, Dona C, Rodríguez-Rosado R, Barreiro P, González-Lahoz J (2000) Discontinuation of secondary prophylaxis for opportunistic infections in HIVinfected patients receiving highly active antiretroviral therapy. AIDS14: 383-386.

14. Benson CA, Kaplan JE, Masur H, Alice Pau, Holmes KK (2004) Treating Opportunistic Infections among HIV-Infected Adults and Adolescents. Recommendations from CDC, the National Institutes of Health, and the HIV Medicine Association/Infectious Diseases Society of America.

15. Manosuthi W, Chaovavanich A, Tansuphaswadikul S, Prasithsirikul W, Inthong Y, et al. (2007) Incidence and risk factors of major opportunistic infections after initiation of antiretroviral therapy among advanced HIV-infected patients in a resource-limited setting. J Infect 55: 464-469.

16. Sun HY, Chen MY, Hsieh SM, Sheng WH, Chang SY, et al. (2006) Changes in the clinical spectrum of opportunistic illnesses in persons with HIV infection in Taiwan in the era of highly active antiretroviral therapy. Jpn J Infect Dis 59: 311-316.

17. Iroezindu MO, Ofondu EO, HauslerH,VanWyk B (2013) Prevalence and Risk Factors for Opportunistic Infections in HIV Patients Receiving Antiretroviral Therapy in a Resource-Limited Setting in Nigeria. J AIDS Clinic Res S3: 002.

18. Mzileni MO, Longo-Mbenza B, Chephe TJ (2008) Mortality and causes of death in HIV-positive patients receiving antiretroviral therapy at Tshepang Clinic in Doctor George Mukhari Hospital. Pol Arch Med Wewn 118: 548-554.

19. Ghate M, Deshpande S, Tripathy S, Nene M, Gedam P, et al. (2009) Incidence of common opportunistic infections in HIV-infected individuals in Pune, India: analysis by stages of immunosuppression represented by CD4 counts. Int J Infect Dis 13: e1-8.

20. Ledergerber B, Egger M, Erard V, Weber R, Hirschel B, et al. (1999) AIDSrelated opportunistic illnesses occurring after initiation of potent antiretroviral therapy: the Swiss HIV Cohort Study. JAMA 282: 2220-2226.

21. Srirangaraj S, Venkatesha D (2011) Opportunistic infections in relation to antiretroviral status among AIDS patients from south India. Indian J Med Microbiol 29: 395-400.

22. Kaplan JE, Hanson D, Dworkin MS, Frederick T, Bertolli J, et al. (2000) Epidemiology of human immunodeficiency virus-associated opportunistic infections in the United States in the era of highly active antiretroviral therapy. Clin Infect Dis 30: 5-14

23. Lawn SD, Badri M, Wood R (2005) Tuberculosis among HIV-infected patients receiving HAART: long term incidence and risk factors in a South African cohort. AIDS 19: 2109-2116.

24. Yazdanpanah Y, Chêne G, Losina E, Goldie SJ, Merchadou LD, et al. (2001) Incidence of primary opportunistic infections in two human immunodeficiency virus-infected French clinical cohorts. Int J Epidemiol 30: 864-871.

25. Lawn SD, Myer L, Bekker LG, Wood R (2006) Burden of tuberculosis in an antiretroviral treatment programme in sub-Saharan Africa: impact on treatment outcomes and implications for tuberculosis control. AIDS 20: 1605-1612. 